\title{
Adding Low Dose hCG to rFSH in GnRH Antagonist ICSI Cycles: A Randomized Controlled Trial
}

\author{
Firas Fadhil Al-Masoody ${ }^{1}$, Manal Taha Al-Obaidi (D) 1
}

${ }^{1}$ High Institute of Infertility Diagnosis and Assisted Reproductive Technologies, Al Nahrain University, Baghdad, Iraq. firas_ffm@yahoo.com

Inhibition of luteinizing hormone (LH) by gonadotropin releasing hormone $(\mathrm{GnRH})$ antagonist may lead to suboptimal response during ovarian stimulation. In addition, several studies suggest that low level of LH is associated with lowered fertilization and implantation rate and increased early pregnancy loss rate. The aim of this study is to study the effect of adding low dose human chorionic gonadotropin (hCG - $200 \mathrm{IU}$ ), as an LH supplement, to recombinant follicle stimulating hormone (rFSH) in a GnRH antagonist cycles in women undergoing in vitro fertilization/intracytoplasmic sperm injection (IVF/ICSI) treatment. Sixty-three infertile women undergoing IVF were randomly divided into two groups. One group was stimulated with the conventional stimulation protocol (rFSH alone), while the second group received $200 \mathrm{IU}$ hCG in addition to rFSH in the late follicular phase (hCG $+\mathrm{rFSH})$. Both groups' results including pregnancy rate, total dose of rFSH required, duration of stimulation, endometrial thickness, oocytes and embryos characteristic, serum hormone levels (Testosterone, Estradiol, Progesterone, and LH) and level of epidermal growth factor (EGF) were compared. The results show that pregnancy rate among the group of women who received the low dose hCG was higher than those who did not receive hCG. However, this difference did not reach statistical significance. Furthermore, other cycle outcomes and hormonal values were comparable between the two stimulation protocols.

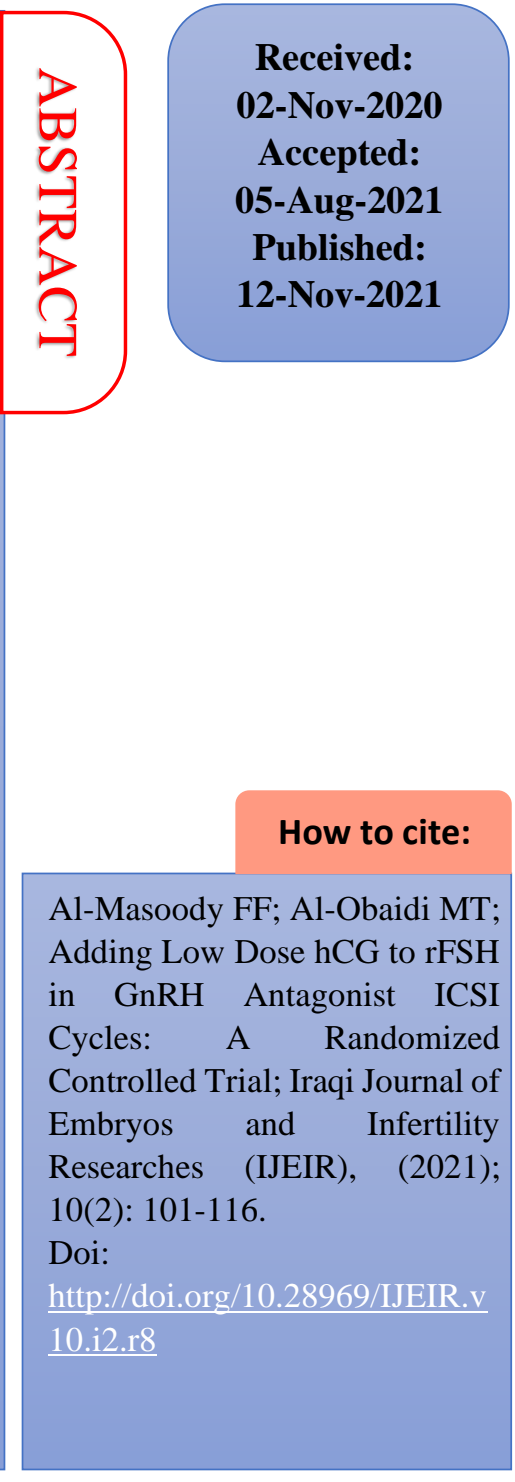

KEYWORDS

GnRH antagonist, Late Follicular Phase, Low Dose hCG, Ovarian Stimulation, LH 


\section{Introduction}

Gonadotropin releasing hormone $(\mathrm{GnRH})$ antagonists are now used more than GnRH agonists in the in vitro fertilization (IVF) stimulation protocols (Efstratios, et al. ${ }^{[1]}$ ). Unlike GnRH agonists which require prolonged period of administration, GnRH antagonists cause immediate suppression of gonadotropin release from the pituitary gland (Tarlatzis, et al. ${ }^{[2]}$ ). Additional benefits include reduction in the dose of recombinant follicle stimulating hormone $(\mathrm{rFSH})$ required for ovarian stimulation, shorter duration of treatment and reduced rate of Ovarian Hyperstimulation Syndrome (OHSS) (Griesinger, G. ${ }^{[3]}$, Khalaf, et al. ${ }^{[4]}$ ). However, GnRH antagonists cause profound suppression of luteinizing hormone (LH) which occurs when the developing follicle is highly sensitive to LH (Griesinger, et al. ${ }^{[5]}$ ). It was found that during GnRH antagonist protocol, the levels of serum LH and estradiol were inversely proportional to the dose of GnRH antagonists (Gardner, et al. ${ }^{[6]}$ ). The two-cell two-gonadotropin theory indicates that both follicle stimulating hormone (FSH) and LH are essential for normal follicle development and steroidogenesis; LH controls the final stages of follicle development, oocyte maturation, ovulation, and support of the corpus luteum; and also stimulates the theca cells to produce androgen, while FSH promotes follicle growth and development and stimulates granulosa cells' aromatase enzyme to convert the androgen produced by the theca cells into oestrogen (Barbieri, RL ${ }^{[7]}$, Hillier, et al. ${ }^{[8]}$ ). Despite the fact that rFSH is the most important gonadotropin, there is a major debate about the importance of adding LH activity to ovarian stimulation protocols used in IVF (Propst, et al. ${ }^{[9]}$ ). Moreover, the minimum threshold level of endogenous LH necessary for normal folliculogenesis is still not known. However, several studies suggested that the low levels of endogenous LH might lead to suboptimal cycle outcomes (Urman, et al. ${ }^{[10]}$ ). It was found that the addition of supplementary $\mathrm{LH}$ to the GnRH antagonist stimulation, lead to higher fertilization rate (Acevedo, et al. ${ }^{[11]}$, Alviggi, et al. ${ }^{[12]}$ ). Likewise, the addition of LH (in the form of low dose of Human Chorionic 
Gonadotropin (hCG)) has been found to promote both follicular growth and oocyte development, and hence lower ampules of rFSH required for ovarian stimulation (Van Horne, et al. ${ }^{[13]}$ ). Nevertheless, other studies showed no significance in the addition of hCG to the GnRH antagonist standard protocol results (Filicori, et al. ${ }^{[14]}$, Serafini, et al. ${ }^{[15]}$ ). Although both regimens are being used nowadays, the controversy of whether hCG should be added to the protocol or not is still standing. Here, we aim to test the hypothesis that the addition of LH supplements in the form of low dose hCG during $\mathrm{GnRH}$ antagonist cycle is beneficial. The primary outcome was to compare the pregnancy rate between two protocols. Other factors including the number of oocytes collected, number of MII oocytes, number of good quality embryos, endometrial thickness, fertilization rate and the required $\mathrm{rFSH}$ dose, were all compared between the two study groups and considered as secondary outcomes. Epidermal growth factor and hormones (estradiol, progesterone, androgen, and LH) were compared between the groups as well.

\section{Materials and Methods}

The target subjects for this study were female patients attending the High Institute for Infertility Diagnosis and Assisted Reproduction Technologies in Al Nahrain University; who were presented for intracytoplasmic sperm injection (ICSI) treatment. Approval for the study was provided by the Local Medical Ethical Committee. All participating individuals signed an informed written consent. The study included 63 women who were selected according to a pre-set inclusion criteria that included; standard indication for ICSI, age between 20 to 40 years old, perceived infertility of more than one year, level of early follicular phase FSH was $<10 \mathrm{mIU} / \mathrm{mL}$, and level of Estradiol was $<60 \mathrm{pg} / \mathrm{mL}$. Excluded cases were women with obesity (having body mass index $(\mathrm{BMI})>30)$, ovarian or uterine pathologies, or endocrine disorders. Besides, any woman with history of poor response to previous IVF cycles or history of OHSS had been eliminated from the study (Ferraretti, et al. $\left.{ }^{[16]}\right)$. Patients were randomly divided into two groups. One group was given hCG (200 
IU at the late follicular phase) in addition to rFSH while the other group did not receive hCG and were stimulated with rFSH alone. All participants first underwent full clinical assessment including medical history, general physical examination, pelvic and transvaginal ultrasound examination, BMI calculation, and hormonal assays at day two of the menstrual cycle. Male partners' seminal fluid was also examined.

\subsection{ICSI Protocol}

Participants went through the GnRH antagonist protocol, as shown in Figure 1. Patients in both groups received 150-300 IU of r-FSH $\quad\left(\right.$ Gonal-F$^{\circledR} \quad$ Serono Laboratories, Germany) subcutaneously starting on day 2 or 3 of the menstrual cycle. When at least two follicles reached $13 \mathrm{~mm}$ in size, $\mathrm{GnRH}$ antagonist (Cetrotide ${ }^{\circledR}$ Serono Laboratories, Inc.) was administered at a dose of $0.25 \mathrm{mg}$ daily until the ovulation trigger day. The initial starting rFSH dose was based on individual's clinical conditions (age, BMI, antral follicle count (AFC)) and previous ovarian response to stimulation. Dose was then adjusted according to patients' response monitored by ultrasound and serum E2 levels. The study group were also given 200 IU daily subcutaneous (SC) injections of hCG (Diclair $^{\circledR}$ HP-hCG, Germany) starting with the Cetrotide ${ }^{\circledR}$ administration. While the control group did not receive the hCG. Pelvic ultrasound examination was carried out to assess the ovaries and endometrial thickness in response to stimulation. Blood samples were collected on the day of ovulation trigger and on day of oocyte pickup (OPU). Afterward, when at least two follicles reached $18 \mathrm{~mm}$, all the previously mentioned drugs were stopped and ovulatory dose of hCG (Ovitrelle ${ }^{\circledR}$, Germany) was given to all patients at a single dose of 2 ampoules of $250 \mu \mathrm{g}$ subcutaneously. Oocyte's pick-up was undertaken 34-36 hours later and ICSI was performed with the husband's sperm. Three to five days later, embryo transfer was performed and luteal support was started.

\subsection{Hormonal Analysis}

Baseline hormone levels were measured at the early follicular phase (day 2 or 3 of the menstrual cycle) which was achieved using 
enzyme linked fluorescent assay (ELFA) technique, via TOSOH analysis equipment. Hormonal assays (LH, progesterone, estradiol, testosterone and epidermal growth factor) were also performed on serum samples collected on the day of ovulation trigger and OPU for patients in the hCG group, while patients in the non-hCG group had their serum checked only on the day of OPU.

\section{Statistical Analysis}

In this prospective randomized controlled trial, statistical analysis was performed using the Statistical Package for the Social Sciences (SPSS) and Microsoft Office Excel 2010. Normality was tested in data sets before the utilization of chi-square and student t-test. Pvalue was set to $<0.05$.

\section{Results}

\subsection{Pregnancy Rate}

The pregnancy rate among the two groups is shown in Table 1. Percentage of pregnancy among patients in the hCG group was higher than in the non-hCG group (37\% vs $16 \%$, respectively).
However, this difference was non-significant statistically speaking $(\mathrm{p}=0.06)$.

\subsection{Hormones' Profile}

Serum levels of $\mathrm{LH}$, progesterone, oestrogen, testosterone, and epidermal growth factor were measured on the day of OPU and compared between the two study groups, as shown in Table 2. Although not statistically significant, the level of E2 was higher in patients who received hCG as compared to those who did not. Serum levels of all other hormones were almost comparable between the two groups of patients.

\subsection{Ovarian Stimulation \\ Characteristics}

The duration of stimulation and the total required dose of rFSH were similar between the two study groups. The average endometrial thickness, measured on the day of ovulation trigger and the day of OPU, among patients who received hCG was higher than those who did not, but this difference did not reach statistical significance, as shown in Table 3. 


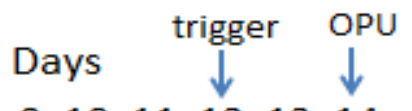

$\begin{array}{lllllllllllll}2 & 3 & 4 & 5 & 6 & 7 & 8 & 9 & 10 & 11 & 12 & 13 & 14\end{array}$

- Non hCG group $\mathrm{rFSH}$

$\mathrm{GnRH}$ antagonist \# 4 flexible based on stimulation response $\begin{array}{lll}0.25 & 0.25 & 0.25\end{array}$

hCG

- hCG group $\mathrm{rFSH}$ $\mathrm{GnRH}$ antagonist 1 flexible based on stimulation response hCG
$\begin{array}{lll}0.25 & 0.25 & 0.25\end{array}$
$200200 \quad 200$

Figure (1): Stimulation protocols using the conventional GnRH antagonist protocol

Table (1): Pregnancy Rate among the study groups

\begin{tabular}{|c|c|c|c|c|}
\hline & $\begin{array}{c}\text { hCG + rFSH } \\
(\mathbf{n = 2 7})\end{array}$ & $\begin{array}{c}\text { rFSH alone } \\
(\mathbf{n = 3 6})\end{array}$ & Total & P value \\
\cline { 1 - 4 } Positive PT & 10 & 6 & 16 & \multirow{2}{*}{0.06} \\
\cline { 1 - 4 } Negative PT & 17 & 30 & 47 & \\
\hline Pregnancy rate & $37 \%$ & $16 \%$ & $25 \%$ & \\
\hline
\end{tabular}

Table (2): Serum hormone levels measured on the day of OPU

\begin{tabular}{|c|c|c|c|}
\hline & $\begin{array}{c}\text { hCG + rFSH } \\
(\mathbf{n = 2 7})\end{array}$ & $\begin{array}{c}\text { rFSH alone } \\
(\mathbf{n = 3 6})\end{array}$ & P value \\
\hline LH (IU/L) & $4.65 \pm 1.25$ & $5.03 \pm 1.84$ & 0.11 \\
\hline Progesterone (ng/ml) & $5.63 \pm 4.18$ & $7.39 \pm 4.07$ & 0.22 \\
\hline Oestrogen (pg/ml) & $1671.1 \pm 953.5$ & $1108.68 \pm 941.87$ & 0.21 \\
\hline Testosterone (pg/ml) & $1.03 \pm 0.51$ & $0.68 \pm 0.49$ & 0.14 \\
\hline EGF (pg/ml) & $47.66 \pm 22.62$ & $74.48 \pm 34.86$ & 0.24 \\
\hline
\end{tabular}

n: number of patients, PT: Pregnancy Test, hCG: human Chorionic Gonadotropin, rFSH: recombinant Follicle stimulating hormone. Mean \pm SD, EGF: Epidermal Growth Factor.

Al-Masoody and Al-Obaidi. http://doi.org/10.28969/IJEIR.v10.i2.r8 
Table (3): Ovarian stimulation characteristics

\begin{tabular}{|c|c|c|c|}
\hline & $\begin{array}{c}\text { hCG + rFSH } \\
(\mathbf{n = 2 7})\end{array}$ & $\begin{array}{c}\text { rFSH alone } \\
(\mathbf{n = 3 6})\end{array}$ & P value \\
\hline rFSH dose (IU) & $1273.66 \pm 236.46$ & $1322 \pm 331$ & 0.47 \\
\hline $\begin{array}{c}\text { Duration of } \\
\text { stimulation } \\
\text { (days) }\end{array}$ & $8.46 \pm 1.37$ & $8.3 \pm 1.4$ & 0.55 \\
\hline $\begin{array}{c}\text { ET (mm) day of } \\
\text { trigger }\end{array}$ & $10.22 \pm 1.67$ & $9.21 \pm 1.2$ & 0.57 \\
\hline $\begin{array}{c}\text { ET (mm) day of } \\
\text { oocyte pick-up }\end{array}$ & $10.81 \pm 1.55$ & $10.03 \pm 1.11$ & 0.56 \\
\hline
\end{tabular}

mm: millimeter

Table (4): Cycle outcomes of all patients in the study

\begin{tabular}{|c|c|c|c|}
\hline & $\begin{array}{c}\text { hCG + rFSH } \\
(\mathbf{n}=\mathbf{2 7})\end{array}$ & $\begin{array}{c}\text { rFSH alone } \\
\mathbf{n}: \mathbf{3 6}\end{array}$ & P value \\
\hline $\begin{array}{c}\text { No. of oocytes } \\
\text { collected }\end{array}$ & $11.32 \pm 4.96$ & $12.73 \pm 6.19$ & 0.34 \\
\hline $\begin{array}{c}\text { No. of mature } \\
\text { oocytes }\end{array}$ & $7.07 \pm 2.82$ & $7.61 \pm 3.09$ & 0.31 \\
\hline $\begin{array}{c}\text { Number of } \\
\text { fertilized oocytes }\end{array}$ & $5.1 \pm 2.64$ & $5.5 \pm 2.88$ & 0.78 \\
\hline $\begin{array}{c}\text { Fertilization rate } \\
\text { per retrieved } \\
\text { oocyte }\end{array}$ & $73 \% \pm 23.27$ & $71 \% \pm 22.45$ & 0.7 \\
\hline $\begin{array}{c}\text { Number of good } \\
\text { quality embryo }\end{array}$ & $2.57 \pm 1.79$ & $2.71 \pm 1.72$ & 0.83 \\
\hline $\begin{array}{c}\text { No. of transferred } \\
\text { embryos }\end{array}$ & $3 \pm 0.94$ & $2.73 \pm 1.15$ & 0.63 \\
\hline
\end{tabular}




\subsection{Cycle Outcomes}

The two groups had almost similar cycle outcomes which included the number of oocytes collected, number of MII oocytes, fertilization rate, number of good embryos and number of transferred embryos, as shown in Table 4.

\section{Discussion}

Nowadays, controlled ovarian stimulation for IVF uses different protocols according to the kind of GnRH analogues (agonist or antagonist) and the gonadotropins (Bosch and Ezcurra ${ }^{[17]}$ ). With regard to gonadotropins, although it is clear that LH plays a crucial role in the natural ovarian cycle, its role in controlled ovarian stimulation and the value of adding it to rFSH is still controversial. Some studies reported that adding $\mathrm{LH}$ to the ovarian stimulation protocols is of benefit in only special group of patients like older women and those with history of poor ovarian response in previous cycle (Mochtar, et al. ${ }^{[18]}$ ), while others suggested that adding $\mathrm{LH}$ to $\mathrm{rFSH}$ during controlled ovarian stimulation has no value (Kolibianakis, et al. ${ }^{[20]}$ ). Both hCG and
LH have $\alpha$ and $\beta$ subunits in their structure; the $\alpha$ subunit is similar, while the $\beta$ subunit is different due to the different glycosylation pattern, which in turn results in different affinity for the $\mathrm{LH} / \mathrm{hCG}$ receptors and different half-life between the two agents (Cole, LA ${ }^{[21]}$ ). Other studies preferred to add low dose hCG to provide LH activity because of the cost effectiveness and the fact that hCG is able to provide the biological activity of $\mathrm{LH}$ at very low doses (Van Horne, et al. ${ }^{[22]}$ ). In ovarian stimulation protocol, it has been shown that the lowest serum level of LH was reached in the late follicular phase as a result of inhibition by the $\mathrm{GnRH}$ analogue. In natural cycle, at this stage of folliculogenesis the granulosa cells $\mathrm{LH}$ receptors increase in the mature follicle as it becomes more dependent on LH than FSH. As a result, it has been proposed that the most appropriate time for adding $\mathrm{LH} / \mathrm{hCG}$ to the stimulation protocol would be the late follicular phase (Filicori, et al. ${ }^{[23]}$ ). We aim here to study the effect of adding $200 \mathrm{IU}$ hCG to $\mathrm{rFSH}$ during the late follicular phase in a GnRH antagonist ICSI cycles. This protocol was compared with women of the same age 
group who were treated with $\mathrm{rFSH}$ alone regimen during GnRH antagonist ICSI cycles.

\subsection{Pregnancy Rate}

The results indicate that pregnancy rate improved by the addition of low dose hCG to ovarian stimulation protocol, although no significant difference was identified. This is consistent with the results of a pervious study which showed that the addition of low dose hCG to stimulation protocols did not have significant beneficial effect on pregnancy rate (Aghahosseini, et al. [24]). Moreover, data from previous research reported that the addition of low dose hCG has no significant beneficial effect in an unselected group of patients (Humaidan, et al. ${ }^{[22]}$ ). The latter study showed a significant improvement in pregnancy rate associated with the addition of LH to the stimulation protocol in women older than 35 years. Hence, including only young patients in this study could be the cause why pregnancy rate was not significantly improved by the addition of low dose hCG. Moreover, some research started low dose hCG simultaneously with rFSH (Propst, et al. [9],
Van Horne, et al. ${ }^{[13]}$ ), while in the current study, hCG was given in the late follicular phase.

\subsection{Required Dose of rFSH}

\section{and Duration of Stimulation}

The total dose of $\mathrm{rFSH}$ required for ovarian stimulation was comparable between the two study groups. Similarly, the duration of stimulation was almost the same. This is in contrast to what had been reported that adding low dose of hCG was associated with less total dose of rFSH and shorter duration of stimulation (Filicori, et al. ${ }^{[23]}$, Serafini, et al. $\left.{ }^{[15]}\right)$. However, in the previous studies, $\mathrm{rFSH}$ was reduced to $75 \mathrm{IU}$ at the start of $\mathrm{hCH}$ administration, whereas in this study, rFSH dose after adding hCG was kept the same.

\subsection{Serum Concentration of}

\section{Estradiol}

Serum level of E2 measured on the day of ovulation trigger and the day of OPU was higher in the hCG group than in the non hCG group. Although this difference did not reach statistical significance, it is consistent with 
previous data (Gomaa, et al. ${ }^{[24]}$ ). Low dose hCG could be the reason behind the rise in serum estradiol concentration. hCG stimulates theca cells androgen production which in turn form the substrate for oestrogen production by the granulosa cells. This direct relation between $\mathrm{LH} / \mathrm{hCG}$ and the rise in serum estradiol has been confirmed in other studies (Levi-Setti, et al. ${ }^{[25]}$, Tarlatzis, et al. ${ }^{[26]}$ ). Moreover, it has been noted that low serum LH level leads to low serum E2 concentration, impaired follicle development and poor reproductive outcomes (Lahoud, et al. ${ }^{[27]}$, O'Dea, et al. ${ }^{[28]}$ ).

\subsection{Other Serum Hormones and Epidermal Growth Factor $(E G F)$}

There was no significant difference in the level of testosterone, $\mathrm{LH}$, or progesterone between the two study groups. Similarly, the level of EGF was comparable between the two study groups. This means that adding LH activity in the form of low dose hCG had no significant effect on any of these hormones measured on the day of OPU.

\subsection{Endometrial Thickness}

(ET)

Adding low dose of hCG to rFSH was not associated with significant increase in the average endometrial thickness both on the day of hCG trigger and pick-up. Tesarik et al. reported that adding $\mathrm{hCG}$ to the stimulation protocol increased the endometrial thickness by an average of $1.5 \mathrm{~mm}$ compared to the group who did not receive hCG (Tesarik, et al. $\left.{ }^{[29]}\right)$. However, it is worth mentioning that this inconsistency could be due to several reasons: the different patients' population, dissimilar interval and dose of hCG, different rate of endometrial growth and also the accuracy of ultrasound measurement.

\subsection{Cycle Outcome}

There was no significant difference in the number of oocytes collected from patients in the two groups. Similarly, the number of mature oocytes (MII), fertilization rate and number of good quality embryos were almost the same between the two groups. This finding 
agrees with the findings from other studies (Serafini, et al. ${ }^{[15]}$ ).

\section{Conclusions}

In $\mathrm{GnRH}$ antagonist protocol, adding low dose of hCG to rFSH in the late stages of follicular development is associated with higher pregnancy rate compared to the rFSH-alone, the difference almost reaches statistical significance $(p=0.06)$. On the other hand, the duration of stimulation, total dose of $\mathrm{rFSH}$ required for stimulation, hormonal profile, endometrial thickness and other oocytes and embryo characteristics were comparable between the two regimens.

\section{Acknowledgment}

We would like to acknowledge Al Nahrain University, Baghdad, Iraq.

\section{Funding}

This work received no funding.

\section{Author Contribution}

Al-Masoody, FF, performed the study, examined and reviewed results, and manuscript writing with the help and supervision of Al-Obaidi, MT.

\section{Conflict of Interest}

The authors declare no conflict of interest.

\section{Ethical Clearance}

The study was approved by the Ethical Approval Committee.

\section{References}

[1]Efstratios M, Kolibianakis JK, Tarlatzis BC. Gonadotropin-releasing hormone antagonists in ovarian stimulation for in vitro fertilization. In Gardner DK, Weissman A, Howles CM, Shoham Z (Eds.), Textbook of Assisted Reproductive Techniques volume 2: Clinical Perspectives, $5^{\text {th }}$ ed., CRC Press, 2018:533-561. [Online article link]

[2] Tarlatzis BC, Fauser BC, Kolibianakis EM, Diedrich K, Devroey P. GnRH antagonists in ovarian stimulation for IVF. Human Reproduction Update. Oxford University Press (OUP); 2006 Mar 24;12(4):333-40. [Online article link]

[3] Griesinger G. Ovarian Hyperstimulation Syndrome Prevention Strategies: Use of 
Gonadotropin-Releasing

Hormone

Antagonists. Seminars in Reproductive

Medicine. Georg Thieme Verlag KG; 2010

Nov;28(06):493-9. [Online article link]

[4] Khalaf OI, Al-Obaidi MT, Al-Anbari LA.

Role of Low-Dose Human Chorionic

Gonadotropin Following Clomiphene

Citrate in Folliculogenesis and Ovulation in Infertile Women. Iraqi Journal of Embryos and Infertility Researches, 2018;8(1):29-37.

\section{[Online article link]}

[5] Griesinger G, Dawson A, Schultze-Mosgau A, Finas D, Diedrich K, Felberbaum R. Assessment of luteinizing hormone level in the gonadotropin-releasing hormone antagonist protocol. Fertility and Sterility. Elsevier BV; 2006 Mar;85(3):791-3. [Online article link]

[6] Gardner DK, Weissman A, Howles CM, Shoham Z. (Eds.). Textbook of Assisted Reproductive Techniques Volume 2: Clinical Perspectives, $5^{\text {th }}$ ed., CRC Press, 2018. [Online article link]

[7]Barbieri RL. The Endocrinology of the Menstrual Cycle. Human Fertility. Springer
New York; 2014;145-69. [Online article link]

[8]Hillier SG, Whitelaw PF, Smyth CD. Follicular oestrogen synthesis: the "twocell, two-gonadotrophin" model revisited. Molecular and Cellular Endocrinology. Elsevier BV; 1994 Apr;100(1-2):51-4. [Online article link]

[9] Propst AM, Hill MJ, Bates GW, Palumbo M, Van Horne AK, Retzloff MG. Low-dose human chorionic gonadotropin may improve in vitro fertilization cycle outcomes in patients with low luteinizing hormone levels after gonadotropin-releasing hormone antagonist administration. Fertility and Sterility. Elsevier BV; 2011 Oct;96(4):898904. [Online article link]

[10] Urman B, Ata B, Yarali H. Endocrine characteristics of assisted reproduction technology cycles. In Gardner DK, Weissman A, Howles CM, Shoham Z. Textbook of Assisted Reproductive Techniques. Volume 2: Clinical Perspectives, $5^{\text {th }}$ ed., CRC Press, 2018:533542. [Online article link] 
[11] Acevedo B, Sanchez M, Gomez JL, Cuadros J, Ricciarelli E, Hernández ER. Luteinizing hormone supplementation increases pregnancy rates in gonadotropinreleasing hormone antagonist donor cycles. Fertility and Sterility. Elsevier BV; 2004 Aug;82(2):343-7. [Online article link]

[12] Alviggi C, Clarizia R, Mollo A, Ranieri A, De Placido G. Who needs LH in ovarian stimulation? Reproductive BioMedicine Online. Elsevier BV; 2011 Feb;22:S33S41. [Online article link]

[13] Van Horne AK, Bates GW, Robinson RD, Arthur NJ, Propst AM. Recombinant follicle-stimulating hormone (rFSH) supplemented with low-dose human chorionic gonadotropin compared with $\mathrm{rFSH}$ alone for ovarian stimulation for in vitro fertilization. Fertility and Sterility. Elsevier BV; 2007 Oct;88(4):1010-3. [Online article link]

[14] Filicori M, Cognigni GE, Gamberini E, Parmegiani L, Troilo E, Roset B. Efficacy of low-dose human chorionic gonadotropin alone to complete controlled ovarian stimulation. Fertility and Sterility. Elsevier
BV; 2005 Aug;84(2):394-401. [Online article link]

[15] Serafini P, Yadid I, Motta E, Alegretti J, Fioravanti J, Coslovsky M. Ovarian stimulation with daily late follicular phase administration of low-dose human chorionic gonadotropin for in vitro fertilization: a prospective, randomized trial. Fertility and Sterility. Elsevier BV; 2006 Oct;86(4):8308. [Online article link]

[16] Ferraretti AP, La Marca A, Fauser BCJM, Tarlatzis B, Nargund G, Gianaroli L. ESHRE consensus on the definition of 'poor response to ovarian stimulation for in vitro fertilization: The Bologna criteria. Human Reproduction, Oxford University Press (OUP); 2011;26(7):1616-1624. [Online article link]

[17] Bosch E, Ezcurra D. Individualised controlled ovarian stimulation (iCOS): maximising success rates for assisted reproductive technology patients. Reproductive Biology and Endocrinology. Springer Science and Business Media LLC; 2011;9(1):82. [Online article link] 
[18] Mochtar MH, Danhof NA, Ayeleke RO, Van der Veen $F$, van Wely $M$. Recombinant luteinizing hormone (rLH) and recombinant follicle stimulating hormone $(\mathrm{rFSH})$ for ovarian stimulation in IVF/ICSI cycles. Cochrane Database of Systematic Reviews. Wiley; 2017 May 24;2017(5). [Online article link]

[19] Kolibianakis EM, Kalogeropoulou L, Griesinger G, Papanikolaou EG, Papadimas $\mathrm{J}$, Bontis $\mathrm{J}$, et al. Among patients treated with FSH and GnRH analogues for in vitro fertilization, is the addition of recombinant LH associated with the probability of live birth? A systematic review and metaanalysis. Human Reproduction Update. Oxford University Press (OUP); 2007 Jun 23;13(5):445-52. [Online article link]

[20] Cole LA. Biological functions of hCG and hCG-related molecules. Reproductive Biology and Endocrinology. Springer Science and Business Media LLC; 2010;8(1):102. [Online article link]

[21] Aghahosseini M, Aleyasin A, Yekehtaz H, Kashani L. Low-dose human chorionic gonadotropin adjunct to an antagonist protocol in assisted reproductive technology: a randomized trial study. Acta Med Iran. 2014;52(6):430-7. PMID: 25130149. [Online article link]

[22] Humaidan P, Bungum M, Bungum L, Yding Andersen C. Effects of recombinant LH supplementation in women undergoing assisted reproduction with $\mathrm{GnRH}$ agonist down-regulation and stimulation with recombinant FSH: an opening study. Reproductive BioMedicine Online. Elsevier BV; 2004 Jan;8(6):635-43. [Online article link]

[23] Filicori M, Cognigni GE, Taraborrelli S, Spettoli D, Ciampaglia W, Tabarelli de Fatis C. Low-dose human chorionic gonadotropin therapy can improve sensitivity to exogenous follicle-stimulating hormone in patients with secondary amenorrhea. Fertility and Sterility. Elsevier BV; 1999 Dec;72(6):1118-20. [Online article link]

[24] Gomaa H, Casper RF, Esfandiari N, Chang P, Bentov Y. Addition of low dose hCG to rFSh benefits older women during ovarian stimulation for IVF. Reproductive 
Biology and Endocrinology. Springer Science and Business Media LLC; 2012 Aug 6;10(1). [Online article link]

[25] Levi-Setti PE, Cavagna M, Bulletti C. Recombinant gonadotrophins associated with GnRH antagonist (cetrorelix) in ovarian stimulation for ICSI: Comparison of r-FSH alone and in combination with r-LH. European Journal of Obstetrics \& Gynecology and Reproductive Biology. Elsevier BV; 2006 Jun;126(2):212-6. [Online article link]

[26] Tarlatzis B, Tavmergen E, Szamatowicz M, Barash A, Amit A, Levitas $\mathrm{E}$, et al. The use of recombinant human $\mathrm{LH}$ (lutropin alfa) in the late stimulation phase of assisted reproduction cycles: a doubleblind, randomized, prospective study. Human Reproduction. Oxford University Press (OUP); 2005 Sep 19;21(1):90-4. [Online article link]

[27] Lahoud R, Jefout MA-, Tyler J, Ryan J, Driscoll G. A relative reduction in midfollicular $\mathrm{LH}$ concentrations during $\mathrm{GnRH}$ agonist IVF/ICSI cycles leads to lower live birth rates. Human Reproduction. Oxford
University Press (OUP); 2006 Jun 19;21(10):2645-9. [Online article link]

[28] O'Dea L, O’Brien F, Currie K, Hemsey G. Follicular development induced by recombinant luteinizing hormone (LH) and follicle-stimulating hormone (FSH) in anovulatory women with LH and FSH deficiency: evidence of a threshold effect. Current Medical Research and Opinion. Informa Healthcare; 2008 Aug 25;24(10):2785-93. [Online article link]

[29] Tesarik J, Hazout A, Mendoza C. Luteinizing hormone affects uterine receptivity independently of ovarian function. Reproductive BioMedicine Online. Elsevier BV; 2003 Jan;7(1):59-64. [Online article link]

Authors at OrcID Manal T. Al-Obaidi https://orcid.org/0000-00018722-3159 


\section{Peer Review Information}

Double-Blind Peer Review in which both authors and reviewers does not know each other.

This work was reviewed by

Asst. Prof. Dr. Hayder A. L. Mossa

Asst. Prof. Dr. Lubna Amer Al-Anbari

Editorial Policy

The editorial policy at IJEIR ensured that

this article fit the standards of scientific

publications.

This work was copyedited by

$\underline{\text { Dr. Taif Alawsi }}$

\section{Authors Biographies}

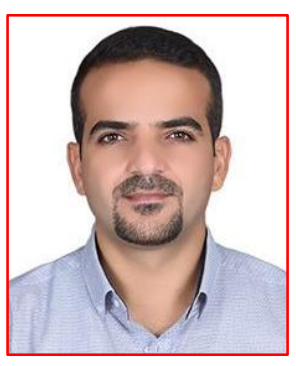

\section{Dr. Firas Fadhil Al-Masoody}

He received his M.B.CH.B. from the College of Medicine, University of Babylon, Babylon, Iraq in 2007. His Master of Medical Sciences in Assisted Reproductive Technologies, from the University of Nottingham, the United Kingdom in 2014. His Ph.D. in Clinical Infertility and Assisted Reproductive Technologies at the High Institute of Infertility Diagnosis and Assisted Reproductive Technologies, Al Nahrain University. He was a rotator in Babylon Health Department until 2011 when he became a Senior doctor in Urology, Al Noor Hospital, Baghdad Health Department. He served as a senior female resident doctor until 2017 at Al-Hilla Teaching Hospital,
Babylon Health Department. Currently, he is an infertility specialist and assisted reproductive technologies, Imam AlSadiq Hospital, Babylon Health Department, Babylon, Iraq.

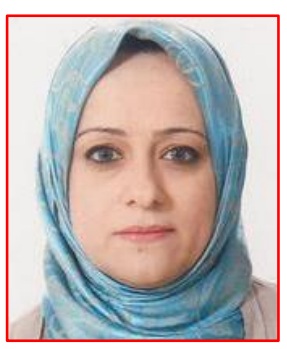

\section{Dr. Manal T. Al-Obaidi}

She received the M.B.CH.B. from AlMustansirya University, the High diploma (equivalent to MSc.) in Assisted Reproductive Technology, Ph.D. in reproductive physiology in 1997, 2003, and 2013 respectively. She occupied several academic positions including the head of the clinical reproductive physiology department and currently, she is the dean of the High Institute of Infertility Diagnosis and Assisted Reproductive Technologies, Al Nahrain University. She published more than 33 articles both local and international.

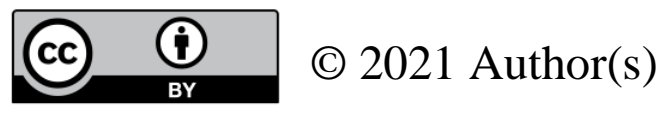

This article is licensed under a Creative Commons Attribution 4.0 International License, which permits use, sharing, adaptation, distribution and reproduction in any medium or format, as long as you give appropriate credit to the original author(s) and the source, provide a link to the Creative Commons license, and indicate if changes were made.

http://creativecommons.org/licenses/by/4.0/. 